\title{
Prevalência de transtornos psiquiátricos em portadores de prótese valvar mecânica com e sem febre reumática
}

\author{
Prevalence of psychiatric disorders in patients with mechanical \\ valve prostheses with and without rheumatic fever
}

Regina Ponce da Silva', Bernardo Rangel Tura², Antonio Egidio Nardi³, Adriana Cardoso de Oliveira e Silva ${ }^{3,4}$

\section{RESUMO}

Objetivo: Valvulopatias cardíacas, causadas por febre reumática e outras etiologias, podem levar ao implante de prótese valvar mecânica e anticoagulação oral obrigatória. Comorbidade psiquiátrica pode reduzir a adesão à anticoagulação. Esse estudo teve como objetivo avaliar a prevalência de transtornos psiquiátricos em pacientes com prótese valvar mecânica. Métodos: Foram avaliados 193 portadores de prótese valvar mecânica, sendo 135 com cardiopatia reumática, tendo sido utilizada a Mini International Neuropsychiatric Interview, versão 5.0.0, para investigação quanto à presença de transtornos psiquiátricos. Resultados: Foram encontrados os seguintes transtornos psiquiátricos nos pacientes avaliados: transtornos de ansiedade generalizada (16,6\%), agorafobia (11,9\%), fobia social $(10,4 \%)$, depressão $(9,8 \%)$, distimia $(4,1 \%$ atual e $1 \%$ no passado), transtorno obsessivo-compulsivo (3,6\%), pânico - vida inteira (1,6\%), dependência ou abuso de substâncias (2\%), dependência ou abuso de álcool (1\%), episódio

\section{Palavras-chave}

Próteses valvulares cardíacas, febre reumática, transtornos psiquiátricos, saúde mental. hipomaníaco (0,5\% atual e 0,5\% no passado), bulimia (0,5\%). O risco de suicídio detectado foi de $13,4 \%$. Ao ser comparada a prevalência dos transtornos no grupo com e sem cardiopatia reumática, não foi encontrada diferença estatisticamente significativa. Conclusão: Foi encontrada elevada comorbidade de transtornos psiquiátricos, principalmente transtornos ansiosos e depressão, sendo também elevado o risco de suicídio nessa população.

\section{ABSTRACT}

Objective: Valvular heart diseases, caused by rheumatic fever and another causes, can lead to implant of mechanical valve prosthesis and mandatory oral anticoagulation. Psychiatric comorbidity may reduce adherence to treatment. This study aimed at assessing the prevalence of psychiatric disorders in patients with mechanical heart valve prostheses. Methods: This study assessed 193 patients with mechanical heart valve prostheses, 135 of whom diagnosed with rheumatic heart disease. The Mini International Neuropsychiatric Interview, version 5.0.0, was used to assess the

\footnotetext{
Trabalho realizado no Instituto Nacional de Cardiologia (INC), Rio de Janeiro (RJ), Brasil. 1 Instituto Nacional de Cardiologia (INC), Serviço de Saúde Mental.

2 Instituto Nacional de Cardiologia (INC), Núcleo de Bioestatística e Bioinformática.

3 Universidade Federal do Rio de Janeiro (UFRJ), Instituto de Psiquiatria, Programa de Pós-graduação em Psiquiatria e Saúde Mental, Laboratório de Pânico e Respiração, INCT Translational Medicine.

4 Universidade Federal Fluminense (UFF), Laboratório de Tanatologia e Psicometria.
} 


\section{Keywords}

Heart valve prosthesis, rheumatic fever, psychiatric disorders, mental health. presence of psychiatric disorders. Results: The following psychiatric disorders were found in the patients with mechanical heart valve prostheses assessed: generalized anxiety disorder (16.6\%); agoraphobia (11.9\%); social phobia (10.4\%); depression (9.8\%); dysthymia (4.1\% current and $1 \%$ past); obsessive-compulsive disorder (3.6\%); panic - lifetime (1.6\%); substance dependence or abuse (2\%); alcohol dependence or abuse (1\%); hypomanic episode (0.5\% current; $0.5 \%$ past); and bulimia (0.5\%). A 13.4\% suicide risk was detected. When comparing the prevalence of psychiatric disorders in the groups with and without rheumatic heart disease, no statistically significant difference was found. Conclusion: High comorbidity of psychiatric disorders, mainly depression and anxiety disorders, was observed in that population, in addition to a high suicide risk.

\section{INTRODUÇÃO}

As doenças cardíacas valvares podem gerar graves complicações e sequelas permanentes. Podem ser causadas por febre reumática (FR) e outras etiologias' ${ }^{1}$ sendo a FR considerada a principal causa de cardiopatia adquirida entre crianças e adultos jovens ${ }^{2}$.

A FR é uma doença inflamatória, com manifestações autoimunes, que ocorre como complicação de infecção primária de orofaringe pelo estreptococo beta-hemolítico do grupo $A^{3}$. Atinge principalmente indivíduos entre 5 e 15 anos, de ambos os sexos, dos quais 30\% a 45\% desenvolvem sequela valvar ${ }^{4}$.

As doenças valvares levam muitos pacientes ao implante de prótese valvar mecânica, que está associada a complicações como trombose local e embolismo ${ }^{5}$, sendo o acidente vascular encefálico uma das suas consequências. Como profilaxia dessas complicações é obrigatória a anticoagulação oral regular e permanente ${ }^{5-9}$, habitualmente feita com varfarina, que reduz em pelo menos quatro vezes a incidência média de tromboembolismo?.

A anticoagulação oral, entretanto, se excessiva, traz risco de sangramentos discretos ou intensos ${ }^{7,9}$, de modo que o fino equilíbrio entre proteção e risco desse tratamento depende criticamente da adesão do paciente ao mesmo, o que nem sempre ocorre. Alguns estudos demonstram que a baixa adesão está na origem da anticoagulação insuficiente, chegando a ser associada a $72,73 \%$ dos resultados terapêuticos não ótimos, com pior prognóstico clínico ${ }^{10}$.

A adesão do paciente à terapêutica medicamentosa pode ser influenciada por comorbidade psiquiátrica"1". O índice de adesão à anticoagulação oral foi estudado em pacientes submetidos a implante de prótese valvar mecânica ${ }^{12}$, mostrando-se inversamente proporcional aos escores de depressão encontrados; os pacientes que apresentavam depressão aderiram significativamente menos que aqueles não deprimidos. Esses resultados indicam o risco que a depressão pode representar para a ocorrência de fenômenos tromboembólicos após implante de prótese valvar mecânica e apontam, em paralelo, para a importância da detecção de transtornos psiquiátricos, por profissional de Saúde Mental, do paciente com indicação de anticoagulação oral por longo prazo.
Considerando o curso crônico e, muitas vezes incapacitante, da FR, que acomete indivíduos jovens em seus anos mais produtivos e ao longo da vida, questionou-se a possibilidade de esses pacientes desenvolverem quadros psiquiátricos com prevalência diferente da do grupo dos não reumáticos.

Não foram encontrados, na literatura, outros trabalhos sobre a ocorrência de transtornos psiquiátricos em portadores de prótese cardíaca valvar mecânica. Esse estudo pretendeu conhecer a prevalência desses transtornos nessa população, buscando ainda verificar se havia associação entre eles e história de FR.

\section{MÉTODO}

Realizou-se um estudo transversal, do qual participaram todos os pacientes que foram atendidos no Ambulatório de Doenças Orovalvares do Instituto Nacional de Cardiologia, no Rio de Janeiro, e que aceitaram participar e preencheram os critérios de pesquisa, entre maio e novembro de 2010, em um total de 193 indivíduos.

Foram adotados os seguintes critérios de inclusão: 1) ser portador de prótese valvar mecânica; 2 ) ter idade igual ou superior a 18 anos completos; 3) mínimo de 6 meses desde a primeira prescrição de anticoagulante oral; 4) aceitar participar do estudo. Os critérios de exclusão foram: 1) presença de qualquer comprometimento que pudesse prejudicar a comunicação do paciente e o livre discurso; 2) desejar retirar-se do estudo. Nenhum indivíduo preencheu os critérios de exclusão.

Para avaliação dos dados referentes à saúde mental, foi utilizada a Mini International Neuropsychiatric Interview $(\mathrm{MINI})^{13}$, versão brasileira 5.0.014, entrevista estruturada desenvolvida para detectar a existência de transtornos psiquiátricos seguindo os critérios do DSM-IV. Foi aplicada por psiquiatra com treinamento para esse fim, cego no momento da entrevista quanto à presença ou ausência de doença reumática.

Foi utilizado questionário elaborado visando manter a padronização das informações colhidas sobre o paciente quanto a seus dados clínicos (presença de prótese mecânica, diagnóstico de doença valvar e tempo de anticoagulação oral) e características de inserção sociodemográica. Os dados foram obtidos por meio de inquérito ao paciente e por meio de informações do prontuário médico. 
Para análise da tabela de contingências, foram utilizados os testes $X^{2}$ e teste exato de Fisher. $O$ teste de normalidade usado foi o de Shapiro-Wilk. Para diferença entre as médias de idades, foi utilizado o teste $t$ de student. Foi adotado nível de significância estatística $p<0,05$.

O presente estudo foi submetido e aprovado pelo Comitê de Ética em Pesquisa da instituição em que foi conduzido. Todos os participantes foram esclarecidos quanto aos objetivos do estudo, seus métodos e a utilização dos dados colhidos, e assinaram o Termo de Consentimento Livre e Esclarecido, conforme determinação da Resolução 196/96 do Conselho Nacional de Saúde.

\section{RESULTADOS}

A população estudada apresentou características sociodemográficas conforme a tabela 1 .

A prevalência de transtornos mentais detectados na amostra total está apresentada na tabela 2.

Dos 193 pacientes que participaram do estudo, 79 (40,3\%) preencheram os critérios para ao menos um transtorno psiquiátrico, tendo sido encontrada elevada prevalência de transtornos ansiosos (transtorno de ansiedade generalizada - TAG, agorafobia, fobia social e transtorno obsessivo-compulsivo) e do humor (depressão e distimia). Destes 79 pacientes, 39 (49,3\%) apresentaram apenas 1 diagnóstico psiquiátrico; 18 (22,8\%) apresentaram 2 transtornos; 10 $(12,6 \%)$ pacientes foram identificados com 3 transtornos; 5 $(6,3 \%)$ pacientes com 4 diagnósticos psiquiátricos e 7 (8,9\%) sujeitos apresentaram 5 diagnósticos combinados.

Depressão atual esteve presente na avaliação de 19 pacientes, sendo não recorrente em 8 pacientes e sendo recorrente em 11 respondentes. O risco de suicídio encontrado foi de 13,4\%. Entre os pacientes sem diagnóstico de FR, 2 (3,4\%) apresentavam alto risco e 5 (8,6\%), baixo risco, totalizando 7 (12\%) pacientes em risco. Já entre os pacientes do grupo com FR, no total $18(13,4 \%)$ estavam em risco, sendo que 4 (3\%) apresentaram alto risco e 14 (10,4\%), baixo risco.
Tabela 2. Prevalência de transtornos psiquiátricos na amostra total

\begin{tabular}{lll}
\hline Transtorno & $\mathbf{n}$ & $(\%)$ \\
\hline Transtorno de ansiedade generalizada & 32 & 16,6 \\
Risco de suicídio & 26 & 13,4 \\
Agorafobia & 23 & 11,9 \\
Fobia social & 20 & 10,4 \\
Depressão & 19 & 9,8 \\
Distimia & 8 & 4,1 \\
Transtorno obsessivo-compulsivo & 7 & 3,6 \\
Dependência/abuso substâncias & 4 & 2,0 \\
Transtorno de pânico - vida inteira & 3 & 1,6 \\
Distimia passado & 2 & 1,0 \\
Dependência/abuso de álcool & 2 & 1,0 \\
Episódio hipomaníaco & 1 & 0,5 \\
Bulimia & 1 & 0,5 \\
\hline
\end{tabular}

O único caso de transtorno hipomaníaco foi encontrado entre os pacientes com FR, com história passada e presente. Também apenas um sujeito apresentou bulimia, no mesmo grupo. Apesar da contraindicação de consumo alcoólico por pacientes em tratamento com anticoagulante oral, dois pacientes apresentaram dependência/abuso de álcool.

Quanto ao diagnóstico de pânico para vida inteira, apresentado por três pacientes, foram encontradas as seguintes comorbidades psiquiátricas: agorafobia, fobia social, TAG e dependência alcoólica em um paciente; agorafobia e TAG em outro paciente e depressão atual e recorrente no terceiro sujeito.

A tabela 3 compara a prevalência de transtornos mentais nos grupos com e sem FR.

Dos 193 pacientes entrevistados, 135 tinham história de FR. Não foi encontrada diferença estatisticamente significativa entre a prevalência de nenhum dos transtornos psiquiátricos investigados nos grupos com e sem FR. Entretanto, observou-se que a maior parte dos pacientes com depressão, risco de suicídio, transtorno obsessivo compulsivo (TOC) ou

Tabela 1. Dados sociodemográficos da amostra total

\begin{tabular}{|c|c|c|c|c|c|c|}
\hline Sexo (\%) & $\begin{array}{c}\text { Homens } 79 \\
(40,9)\end{array}$ & $\begin{array}{l}\text { Mulheres } \\
114(59,1)\end{array}$ & $\begin{array}{l}\text { Total } \\
193\end{array}$ & & & \\
\hline Idade (SD) & $\begin{array}{c}\text { Homens } \\
52,9( \pm 13,1)\end{array}$ & $\begin{array}{c}\text { Mulheres } \\
53,0( \pm 11,1)\end{array}$ & $\begin{array}{c}\text { Total } \\
53,9( \pm 11,9)\end{array}$ & & & \\
\hline Estado civil (\%) & $\begin{array}{c}\text { Casados } \\
125(64,8)\end{array}$ & $\begin{array}{c}\text { Separados } \\
18(9,3)\end{array}$ & $\begin{array}{l}\text { Solteiros } \\
28(14,5)\end{array}$ & $\begin{array}{c}\text { Viúvos } \\
22(11,4)\end{array}$ & & \\
\hline Etnia (\%) & $\begin{array}{c}\text { Brancos } \\
129(66,8)\end{array}$ & $\begin{array}{l}\text { Pardos } \\
51(26,4)\end{array}$ & $\begin{array}{l}\text { Negros } \\
13(6,7)\end{array}$ & & & \\
\hline Ocupação atual (\%) & $\begin{array}{c}\text { Empregados } \\
62(32,1)\end{array}$ & $\begin{array}{c}\text { Aposentados } \\
68(35,2)\end{array}$ & $\begin{array}{l}\text { Do lar } \\
46(23,8)\end{array}$ & $\begin{array}{l}\text { Licença } \\
12(6,2)\end{array}$ & $\begin{array}{c}\text { Estudantes } \\
3(1,6)\end{array}$ & $\begin{array}{c}\text { Sem emprego } \\
2(1,0)\end{array}$ \\
\hline Religião (\%) & $\begin{array}{l}\text { Católica } \\
88(45,6)\end{array}$ & $\begin{array}{l}\text { Evangélica } \\
73(37,8)\end{array}$ & $\begin{array}{l}\text { Outras } \\
15(7,8)\end{array}$ & $\begin{array}{c}\text { Nenhuma } \\
17(8,8)\end{array}$ & & \\
\hline
\end{tabular}


Tabela 3. Comparação entre pacientes com e sem febre reumática para presença de transtornos psiquiátricos

\begin{tabular}{lccc}
\hline & $\begin{array}{c}\text { Com febre } \\
\text { reumática (\%) }\end{array}$ & $\begin{array}{c}\text { Sem febre } \\
\text { reumática (\%) }\end{array}$ & Valor dep \\
\hline Episódio depressivo maior atual & 11,9 & 5,2 & 0,15 \\
Episódio depressivo maior recorrente & 6,7 & 3,4 & 0,37 \\
Episódio depressivo maior com & 2,2 & 0,0 & 0,25 \\
características melancólicas & & & \\
Distimia atual & 3,0 & 6,9 & 0,20 \\
Distimia passado & 0,0 & 1,7 & 0,12 \\
Risco de suicídio alto & 21,1 & 28,6 & 0,68 \\
Risco de suicídio baixo & 78,9 & 71,4 & 0,68 \\
Episódio maníaco atual & 0,7 & 0,0 & 0,51 \\
Transtorno de pânico - vida inteira & 1,5 & 1,7 & 0,90 \\
Agorafobia & 11,1 & 13,8 & 0,59 \\
Fobia social & 11,1 & 8,6 & 0,60 \\
Transtorno obsessivo-compulsivo & 4,4 & 1,7 & 0,35 \\
Dependência/abuso de álcool & 0,7 & 1,7 & 0,53 \\
Dependência/abuso de substâncias & 1,5 & 3,4 & 0,37 \\
Bulimia & 0,7 & 0,0 & 0,51 \\
Transtorno de ansiedade generalizada & 17,0 & 15,5 & 0,79 \\
\hline
\end{tabular}

TAG, bem como os que apresentaram 5 diagnósticos associados, tinha história de FR. Não se pode descartar a possibilidade de que um estudo com amostra maior mostre valor de p significativo na comparação entre os dois grupos.

Com respeito à relação entre presença de transtornos mentais na amostra total estudada e variáveis sociodemográficas, algumas observações foram feitas.

Na relação entre presença de transtornos mentais e sexo, foi observada uma maior prevalência de depressão em muIheres $(8,8 \%)$ do que em homens $(1,3 \%)$, com $p=0,027$. No TAG, a prevalência no sexo feminino também foi maior que no masculino $(21,1 \%$ contra $10,1 \%, p=0,045)$. As demais associações não alcançaram significância estatística.

Em relação à etnia, foi observada uma diferença significativa para episódio depressivo maior com características melancólicas $(p=0,014)$ com predomínio em pardos e para TAG $(p=0,048)$ com predomínio em brancos.

Quanto à religião, registrou-se diferença significativa para risco de suicídio ( $p=0,030)$, sendo todos os casos classificados como alto risco presentes em sujeitos evangélicos, que tiveram também 5 registros de baixo risco; entre católicos foi detectado risco de suicídio classificado como baixo em 11 pacientes.

Quanto à idade, observou-se diferença significativa em relação à dependência/abuso de substâncias não alcoólicas ( $p=0,015$ ), tendo média etária 67,25 os pacientes que se enquadraram nessa categoria, enquanto os demais apresentaram média de 52,67 anos.

A tabela 4 mostra a comparação entre os grupos de pacientes com e sem FR para algumas variáveis sociodemográficas.
Tabela 4. Comparação entre pacientes com e sem febre reumática para variáveis socioeconômicas e culturais

\begin{tabular}{|c|c|c|}
\hline & Com febre reumática & Sem febre reumática \\
\hline \multicolumn{3}{|l|}{$\operatorname{Sexo}(p=0,00)$} \\
\hline Feminino & $97(71,8 \%)$ & $17(29,3 \%)$ \\
\hline Masculino & $38(28,1 \%)$ & $41(70,6 \%)$ \\
\hline Idade $(p=0,00)$ & $\begin{array}{l}52,4( \pm 12,0) \\
\text { Mínimo = 18; } \\
\text { Máximo = } 80\end{array}$ & $\begin{array}{c}54,1( \pm 11,6) \\
\text { Mínimo }=23 \\
\text { máximo }=80\end{array}$ \\
\hline \multicolumn{3}{|l|}{ Estado civil $(p=0,55)$} \\
\hline Casado & $83(61,5 \%)$ & $42(72,4 \%)$ \\
\hline Divorciado/separado/desquitado & $14(16,6 \%)$ & $4(6,8 \%)$ \\
\hline Solteiro & $22(16,3 \%)$ & $6(10,3 \%)$ \\
\hline Viúvo & $16(11,9 \%)$ & $6(10,3 \%)$ \\
\hline \multicolumn{3}{|l|}{ Ocupação $(p=0,00)$} \\
\hline Desempregado & $1(0,7 \%)$ & $1(1,7 \%)$ \\
\hline Do lar & $41(30,4 \%)$ & $5(8,6 \%)$ \\
\hline Aposentado & $38(28,1 \%)$ & $30(51,7 \%)$ \\
\hline Estudante & $3(2,2 \%)$ & $0(0,0 \%)$ \\
\hline Empregado & $44(32,5 \%)$ & $18(31,0 \%)$ \\
\hline Licença & $8(5,9 \%)$ & $4(6,9 \%)$ \\
\hline \multicolumn{3}{|l|}{ Etnia $(p=0,90)$} \\
\hline Brancos & $89(65,9 \%)$ & $40(69 \%)$ \\
\hline Pardos & $36(26,7 \%)$ & $15(25,9 \%)$ \\
\hline Negros & $10(7,4 \%)$ & $3(5,2 \%)$ \\
\hline \multicolumn{3}{|l|}{ Religião $(p=0,54)$} \\
\hline Católica & $63(46,7 \%)$ & $25(43,1 \%)$ \\
\hline Evangélica & $52(38,5 \%)$ & $21(36,2 \%)$ \\
\hline Outras & $8(5,8 \%)$ & $7(12 \%)$ \\
\hline Não tem religião & $12(8,9 \%)$ & $5(8,6 \%)$ \\
\hline \multicolumn{3}{|l|}{ Escolaridade $(p=0,37)$} \\
\hline Sem educação formal & $4(3 \%)$ & $0(0,0 \%)$ \\
\hline Primeiro Grau & $91(67,4 \%)$ & $45(77,6 \%)$ \\
\hline Segundo Grau & $34(25,2 \%)$ & $10(17,2 \%)$ \\
\hline Superior & $6(4,4 \%)$ & $3(5,2 \%)$ \\
\hline \multicolumn{3}{|l|}{ Tempo de anticoagulação $(p=0,64)$} \\
\hline Menos de 1 ano & $8(5,9 \%)$ & $7(12,1 \%)$ \\
\hline 1 a 5 anos & $67(49,6 \%)$ & $27(46,6 \%)$ \\
\hline 6 a 10 anos & $34(25,2 \%)$ & $17(29,3 \%)$ \\
\hline 11 a 15 anos & $12(8,9 \%)$ & $4(6,9 \%)$ \\
\hline 16 a 20 anos & $6(4,4 \%)$ & $1(1,7 \%)$ \\
\hline Mais de 20 anos & $8(5,9 \%)$ & $2(3,4 \%)$ \\
\hline
\end{tabular}

Para o estudo da relação entre presença ou ausência de diagnóstico de FR e as variáveis, foram utilizados os testes do $X^{2}$ para as variáveis sexo, etnia e tempo de anticoagulação; para as variáveis estado civil, ocupação atual, religião e escolaridade foi usado o teste exato de Fisher. Para a variável idade, foi aplicado o t student. Observou-se significância estatística para as variáveis sexo, idade e ocupação atual, sendo os grupos comparáveis. 


\section{DISCUSSÃO}

A predominância de transtornos encontrados neste trabalho está em acordo com estudo multicêntrico ${ }^{15}$ que verificou maior prevalência de transtornos de ansiedade na população estudada, seguidos por transtornos fóbicos. Em nossa pesquisa, o TAG teve a mais alta prevalência na amostra total, também em acordo com os resultados encontrados em revisão sistemática de 1997 a 2009¹6, na qual o transtorno de ansiedade apareceu em primeiro lugar na população adulta brasileira, seguido dos estados fóbicos e depressivos.

Os episódios de depressão maior ocorreram em 9,8\% da amostra total, resultado congruente com estudos ${ }^{17}$ que registram prevalência anual na população geral com variação entre $3 \%$ e $11 \%$. Entretanto, se analisados em conjunto com os casos de distimia, os quais, segundo literatura ${ }^{17}$, trazem maior prejuízo funcional que episódios de depressão maior, alcançam prevalência de 13,98\%, índice elevado de transtornos de humor que pode favorecer a baixa adesão à anticoagulação oral por essa população. O histórico individual de cardiopatia crônica, cursando com hospitalizações, limitações da vida diária e contato muitas vezes próximo com a percepção de finitude, poderia estar relacionado ao índice elevado desses transtornos no grupo estudado.

$\mathrm{Na}$ amostra estudada, a prevalência do risco de suicídio foi bastante superior à da população geral, estimada entre $1,8 \%$ e $3,6 \%{ }^{18}$.

A prevalência de TOC encontrada em nosso estudo foi de 3,6\% na amostra total, sem diferença estatisticamente significativa entre os grupos com e sem FR. Estudo anterior ${ }^{19}$ indicou maior prevalência de TOC entre os indivíduos com história de FR. Essa diferença entre os resultados dos dois estudos pode estar relacionada ao fato de a MINI pesquisar apenas o TOC e não abordar os sintomas obsessivo-compulsivos nem tiques, que foram incluídos no estudo dos outros autores.

Sobre a fobia social (quarto transtorno mais prevalente no grupo estudado, afetando 10,4\% dos pacientes), apesar de não existirem na literatura estudos específicos sobre sua presença em portadores de prótese valvar, sabe-se que, em coronariopatas, o isolamento social, característico de sujeitos que apresentam esse quadro psiquiátrico, apresenta relação com menor sobrevivência ${ }^{20}$.

Quanto ao espectro de pânico, foram encontrados 23 casos de agorafobia sem história de transtorno de pânico atual $(11,9 \%)$ e apenas 3 casos de transtorno de pânico de vida inteira. Não foi encontrado qualquer caso de transtorno de pânico atual. Tal fenômeno poderia encontrar explicação em uma variante do transtorno de pânico caracterizada pelos ataques sem associação de medo. Pacientes que apresentam transtorno de pânico sem medo 21,22 (TPSM) responderiam por 32\% a $41 \%$ dos pacientes com transtorno de pânico que buscam tratamento médico em decorrência de episódios de dor torácica²1.
A MINI tem, como foco inicial para o diagnóstico de transtorno de pânico, a ansiedade, o desconforto e o medo. Apenas os pacientes que respondem positivamente a esse critério passam para os itens seguintes, que apresentam os sinais e sintomas físicos característicos do ataque de pânico. Desse modo, pacientes que se enquadrem na categoria TPSM teriam um resultado negativo para presença de pânico.

Pacientes TPSM, segundo estudo comparativo realizado com pacientes com diagnóstico de transtorno de pânico e sem transtorno de pânico ${ }^{23}$, na comparação com o segundo grupo, apesar de apresentarem escores inferiores para presença de dor torácica no último mês (50\% versus 60\%) e busca por serviços de emergência (27\% versus $47 \%$ ), tiveram maior índice de hospitalização (35\% versus 30\%). Desses pacientes, 31\% TPSM percebiam a causa de seu desconforto como de origem cardíaca, enquanto apenas 7\% dos pacientes TP o faziam.

Futuros estudos devem ser realizados com utilização de outros instrumentos, que sejam capazes de identificar a presença dessa categoria específica de pânico, dada sua importância e a ainda presente controvérsia quanto à associação entre transtorno de pânico e valvulopatias ${ }^{24}$, nessa população específica.

Apesar de terem sido registrados quatro casos na categoria "dependência/abuso de substâncias", ao se avaliar quais substâncias haviam sido utilizadas, encontraram-se como resposta diazepam para dois pacientes e midazolam e lorazepam para os outros dois. Deve-se considerar que tais fármacos foram introduzidos na rotina desses pacientes mediante prescrição médica e fazem parte do plano terapêutico deles. Isso justifica a resposta sim ao item "(...) durante os últimos 12 meses, usou várias vezes uma dessas substâncias para se sentir melhor (...)" assim como o fato de serem psicofármacos colabora para a afirmativa "(...) para mudar seu estado de humor". Como a MINI não busca esclarecer se o aumento do consumo da substância ocorreu por iniciativa do paciente ou se atendeu a orientação médica de aumento da dose, o termo "dependência/abuso" pode não se adequar ao consumo de fármacos realizado por esses pacientes.

As taxas de prevalência encontradas nesse estudo poderiam ser ainda maiores se considerados conjuntos de sinais e sintomas, ou até mesmo síndromes ansiosas ou depressivas. Por meio do instrumento adotado nesse estudo, no entanto, foi avaliada apenas a existência de transtornos, sendo necessárias investigações futuras para complementar os dados aqui encontrados, principalmente visando a políticas de profilaxia em saúde mental, intervindo nos casos em que são detectados problemas de saúde mental em suas manifestações iniciais.

Outra limitação do estudo foi a ausência de avaliação de sintomas subclínicos, não considerados no método utilizado, bem como o pequeno tamanho amostral e a transversalidade da pesquisa. 
A alta prevalência de transtornos psiquiátricos nessa população gera questionamento quanto a estarem ou não recebendo atendimento adequado para essa demanda. Cabe informar que, aos pacientes que preencheram critérios para diagnóstico de algum transtorno, foi oferecido, logo após a entrevista, encaminhamento para tratamento psiquiátrico.

\section{CONCLUSÃO}

A pesquisa de transtornos psiquiátricos em portadores de prótese valvar mecânica revelou elevada prevalência de transtornos ansiosos, depressão e risco de suicídio, realçando a importância da detecção e tratamento dos transtornos mentais nessa população.

A presença de FR não alterou significativamente os valores encontrados para prevalência dos diversos transtornos mentais nesses pacientes.

Sugerimos novos estudos, mais detalhados quanto a sintomas subclínicos e quanto à comorbidade apresentada entre os diversos transtornos mentais encontrados, assim como avaliação da correlação entre quadros psiquiátricos e variáveis como sítio e número de próteses, tempo de uso do anticoagulante, uso de outros medicamentos, história de acidente vascular encefálico e história de coreia de Sydenham.

\section{REFERÊNCIAS}

1. Guglielmi L, Kalil J. Febre reumática: conhecimento atual da etiopatogenia. In: Timerman A, Machado César LA, Ferreira JFM, Bertolami MC. Manual de Cardiologia; SOCESP. São Paulo: Atheneu; 2000. p. 265-70.

2. World Health Organization (WHO). Rheumatic fever and rheumatic heart disease: report of a WHO Expert Consultation, Geneva, 29 October - 1 November 2001. WHO Technical Report Series; 923.

3. Terreri MTRA, Caldas AM, Len CA, Ultchak F, Hilário MOE. Características clínicas e demográicas de 193 pacientes com febre reumática. Rev Bras Reumatol. 2006;46:385-90.

4. Lima ABR, Barros M, Santos M. Descrição dos indicadores sociais em adolescentes portadores de febre reumática. Revista da SOCERJ. 2005;18:30-5.

5. Bonow RO, Carabello BA, Chatterjee K, de Leon AC Jr., Faxon DP, Freed MD, et al. 2008 focused update incorporated into the ACC/AHA 2006 guidelines for the management of patients with valvular heart disease: a report of the American College of Cardiology/American Heart Association Task Force on Practice Guidelines (Writing Committee to Develop Guidelines for the Management of Patients With Valvular Heart Disease). J Am Coll Cardiol. 2008;52:e1-142.

6. Salem DN, Stein PD, Al-Ahmad A, Bussey HI, Horstkotte D, Miller N, et al. Antithrombotic Therapy in valvular heart disease--native and prosthetic: the Seventh ACCP Conference on Antithrombotic and Thrombolytic Therapy. Chest. 2004;126:457-82.
7. Cannegieter SC, Rosendaal FR, Briët E. Thromboembolic and bleeding complications in patients with mechanical heart valve prostheses. Circulation. 1994;89(2):635-41.

8. Sacco RL, Adams R, Albers G, Alberts MJ, Benavente 0, Furie K, et al. Guidelines for Prevention of Stroke in Patients With Ischemic Stroke or Transient Ischemic Attack: a statement for healthcare professionals from the American Heart Association/American Stroke Association Council on Stroke. Stroke. 2006;37:577-617.

9. Hirsh J, Fuster V, Ansell J, Halperin JL; American Heart Association/American College of Cardiology Foundation. American Heart Association/American College of Cardiology Foundation Guide to Warfarin Therapy. Circulation. 2003;107(12):1692-711.

10. Kimmel SE, Chen Z, Price M, Parker CS, Metlay JP, Christie JD, et al. The Influence of Patient Adherence on Anticoagulation Control With Warfarin: Results From the International Normalized Ratio Adherence and Genetics (IN-RANGE) Study. Arch Intern Med. 2007;167(3):229-35.

11. World Health Organization (WHO). Adherence to long-term therapies: Evidence for action. Geneva: WHO; 2003.

12. El-Gatit AS, Haw M. Relationship between depression and non-adherence to anticoagulant therapy after valve replacement. East Mediterr Health J. 2003;9(1-2):12-9.

13. Sheehan DV, Lecrubier Y, Sheehan KH, Amorim P, Janavs J, Weiller E, et al. The Mini-International Neuropsychiatric Interview (M.I.I.I.I): the development and validation of a structured diagnostic psychiatric interview for DSM-IV and ICD-10. J Clin Psychiatry. 1998;59 Suppl 20:22-33; quiz 34-57.

14. Amorim P. Mini-International Neuropsychiatric Interview (MINI): validação de entrevista breve para diagnóstico de transtornos mentais. Rev Bras Psiquiatr. 2000;22(3):106-15.

15. Mello MF, Mello AAF, Kohn R, orgs. Epidemiologia da saúde mental no Brasil. Porto Alegre: Artmed; 2007.

16. Santos EG, Siqueira MM. Prevalência dos transtornos mentais na população adulta brasileira: uma revisão sistemática de 1997 a 2009. J Bras Psiquiatr. 2010;59(3):238-46.

17. Fleck MP, Berlim MT, Lafer B, Sougey EB, Del Porto JA, Brasil MA, et al. Revisão das diretrizes da Associação Médica Brasileira para o tratamento da depressão. Rev Bras Psiquiatr. 2009;31(Supl I):S7-17.

18. Botega NJ, Barros MBA, Oliveira HB, Dalgalarrondo P, Marin-León L. Suicidal behavior in the community: Prevalence and factors associated with suicidal ideation. Rev Bras Psiquiatr. 2005;27(1):45-53.

19. Alvarenga PG, Hounie AG, Diniz JB, Salem M, Spina G, Miguel EC. Obsessive-Compulsive Symptoms in Heart Disease Patients With and Without History of Rheumatic Fever. J Neuropsychiatry Clin Neurosci. 2006;18(3):405-8.

20. Brummet BH, Barefoot JC, Siegler IC, Clapp-Channing NE, Lytle BL, Bosworth HB, et al. Characteristics of socially isolated patients with coronary artery disease who are at elevated risk for mortality. Psychosom Med. 2001;63(2):267-72.

21. Beitman BD, Basha I, Flaker G, DeRosear L, Mukerji V, Lamberti J. Non-fearful panic disorder: panic attacks without fear. Behav Res Ther. 1987;25(6):487-92.

22. Beitman BD, Kusher M, Lamberti JW, Mukerji V. Panic Disorder without fear in patients with angiographically normal coronary arteries. J Nerv Ment Dis. 1990;178(5):307-12.

23. Fleet RP, Martel JP, Lavoie KL, Dupuis G, Beitman BD. Non-fearful Panic Disorder: a variant of panic in medical patients? Psychosomatics. 2000;41(4):311-20.

24. Filho AS, Maciel BC, Matin-Santos R, Romano MMD, Crippa JA. Does the association between mitral valve prolapse and panic disorder really exist? Prim Care Companion J Clin Psychiatry. 2008;10(1):38-47. 